\title{
Critical Behavior of a Bounded Kardar-Parisi-Zhang Equation
}

\author{
Miguel A. Muñoz, \\ Departamento de Electromagnetismo y Física de la Materia, Universidad de Granada, Fuentenueva s/n, 18071 Granada, Spain \\ Francisco de los Santos, \\ Center for Polymer Studies and Department of Physics, Boston University, Boston, MA 02215, USA \\ and Abdelfattah Achahbar \\ Departement de Physique, Faculté des Sciènces, B.P. 2121 M'hannech, 93002 Tetouan, Morocco
}

Received on 1st April, 2003

\begin{abstract}
A host of spatially extended systems, both in physics and in other disciplines, are well described at a coarsegrained scale by a Langevin equation with multiplicative-noise. Such systems may exhibit nonequilibrium phase transitions, which can be classified into universality classes. Here we study in detail one such class that can be mapped into a Kardar-Parisi-Zhang (KPZ) interface equation with a positive (negative) non-linearity in the presence of a bounding lower (upper) wall. The wall limits the possible values taken by the height variable, introducing a lower (upper) cut-off, and induces a phase transition between a pinned (active) and a depinned (absorbing) phase. This transition is studied here using mean field and field theoretical arguments, as well as from a numerical point of view. Its main properties and critical features, as well as some challenging theoretical difficulties, are reported. The differences with other multiplicative noise and bounded-KPZ universality classes are stressed, and the effects caused by the introduction of "attractive" walls, relevant in some physical contexts, are also analyzed.
\end{abstract}

\section{Introduction}

Nonequilibrium phase transitions occurring in systems amenable to be described by Langevin equations including a multiplicative noise $(\mathrm{MN})$ term are the subject of current intense studies. This embraces a broad variety of systems both in physics and in other disciplines. A phenomenology much richer and complex than that appearing in equilibrium systems, including counterintuitive behaviors, has been reported to appear in these, typically nonequilibrium, situations. See $[1,2]$ for detailed introductions to this growing field, including many different realizations.

The interest in MN problems is enlarged even further, because of the existing mappings between them and other prototypical nonequilibrium problems [2]. A well known instance is the Kardar-Parisi-Zhang (KPZ) equation, describing the kinetic roughening transition of generic interfaces under nonequilibrium conditions [3, 5, 4], which can be mapped onto a MN Langevin equation, by performing the so called Cole-Hopf transformation linking the interface height at each point with the activity field of the MN equation. If an interface under consideration is described by the KPZ equation and it is physically limited by a wall, i.e. if the heights cannot be larger or smaller than a certain value, then this problem, bounded $\mathrm{KPZ}$, can be mapped into a multiplicative noise equation by employing the abovementioned transformation $[2,6,7]$. The bounded KPZ equation may experience, as parameters are varied, a phase transition from a depinned phase in which the interface escapes with probability one from the wall, to a pinned phase characterized by a finite expectation value of the stationary averaged height (measured from the wall). In the MN language (i.e. after employing the Cole-Hopf transformation) the pinning-depinning transition corresponds to a critical point separating an absorbing phase in which the order parameter goes exponentially to zero (depinned phase) to an active phase in which the order parameter takes a nonvanishing average value.

Surprisingly enough, it was shown a few years ago that the introduction of "upper" or "lower" walls into a given KPZ equation (with a fixed non-linearity sign) lead to quite different phenomenologies. The origin of this can be tracked down to the fact that the KPZ equation is not invariant upon inverting the height (see [8] or [2] for a more detailed explanation). Taking, for instance, the sign of the coefficient of the KPZ non-linearity to be positive, the introduction of an upper wall leads to a (well established by now) set of critical exponents characterizing the so called multiplicative noise 1 (MN1) universality class [6-9]. On the other hand, a wall limiting negative values of the interface height (lower wall) leads to a different type of phase transition as shown by $\mathrm{T}$. Hwa and one of us some years back [8]. In what follows, and following the nomenclature introduced in [2], we will use the term MN2 to denote this class.

It can be easily shown that a KPZ equation with positive 
non-linearity and a lower wall is completely equivalent to a KPZ with a negative non-linearity coefficient and an upper wall [2]: one just has to change the sign of the height variable in the KPZ-like equation to verify this.

The MN2 class is of great importance in the context of alignment of DNA and other biological sequences. It has been argued by Hwa and collaborators, that the phase transition appearing upon changing the so called scoring parameter in the commonly used alignment algorithm can be mapped into the MN2 critical point [14]. It is also related to some instances of nonequilibrium wetting [10]. For some other applications and physical instances within this class see [2] and references therein.

While the MN1 class has been extensively studied, especially after its connections with nonequilibrium wetting [10-12] and with the problem of synchronization in extended systems were established $[2,13]$, the MN2 class remains poorly studied. Furthermore, recent numerical analyses have revealed that the preliminary critical exponent values reported in [14] might be far from their true asymptotic values.

Aimed at clarifying these issues, it is the purpose of this paper to analyze the MN2 phase transition in one dimensional systems using: i) mean-field and field-theoretical techniques and ii) numerical (Monte Carlo) analysis of different models claimed to belong to this class.

Finally let us stress that if the wall becomes attractive, rather than simply bounding, new phenomenology might appear. This is particularly interesting in the context of synchronization $[2,13]$. This possibility will also be discussed in the paper.

\section{The MN2 class}

Let us consider a KPZ equation with a positive non-linearity coefficient, $\lambda>0$, in the presence of a lower wall,

$$
\partial_{t} h(x, t)=a+b e^{-p h}+D \nabla^{2} h+\lambda(\nabla h)^{2}+\sigma \eta(x, t) .
$$

where $h$ is a height variable, $a$ represents a constant drift while $b e^{-p h}$ is a bounding wall. The parameter $p>0$ controls the wall penetrability, the limit $p \rightarrow \infty$ corresponding to a perfectly rigid (impenetrable) wall. It has been shown previously that for both the MN1 and the MN2 classes the magnitude of $p$ does not influence the asymptotic properties at criticality $[6,7]$ (its sign, however, is important, as it determines whether the wall is a lower or an upper one). The same property applies to equilibrium systems (i.e., for $\lambda=0)$ [15]. $\eta$ is a stochastic white noise with $\langle\eta\rangle=0$ and $\left\langle\eta(x, t) \eta\left(x^{\prime}, t^{\prime}\right)\right\rangle=2 \delta\left(t-t^{\prime}\right) \delta\left(x-x^{\prime}\right)$, where $\langle\cdot\rangle$ denotes an average over the distribution of the noise.

For a fixed value of $b$ the interface experiences a pinningdepinning transition at some value $a=a_{c}$.

Some remarks concerning the connection of the previous equation with wetting problems follow. When $h(x, t)$ is viewed as the distance separating a liquid-gas interface from a solid wall, Eq. (1) can then be interpreted as a dynamic model for nonequilibrium wetting. Under this perspective $a$ is the chemical potential difference between the liquid and the gas phases, $\langle h\rangle$ is the thickness of the wetting layer, and the wall is a, physical substrate. At bulk phase coexistence, i.e. for the value of $a=a_{c}$ for which in the absence of the wall the interface does not move on average, $\langle h\rangle$ diverges at all temperatures above certain wetting temperature, $T_{W}$, while for $a \neq a_{c}$ the thickness of the liquid film can be big, but finite (pinned interface). The temperature here is controlled by the parameter $b$, which vanishes linearly with the mean-field wetting temperature as $T-T_{W}$. Thus, on approaching coexistence for $T>T_{W}(b>0$ at the meanfield level), $\langle h\rangle$ diverges as $\langle h\rangle \sim\left|a-a_{c}\right|^{\beta_{h}}$. This transition, termed complete wetting, is always continuous and the value of the $\beta_{h}$ exponent depends on the nature of the forces between the particles in the fluid phases and the wall. In this paper only short-range, exponentially decaying interactions between all the particles and the substrate (as described by Eq. (1)) are considered.

The change of variables $n=\exp (-h)$ transforms Eq. (1) into the MN2 Langevin equation:

$$
\partial_{t} n(x, t)=\nabla^{2} n-2 \frac{(\nabla n)^{2}}{n}-(a+1) n-b n^{p+1}+n \eta
$$

where for the sake of simplicity we have set $\lambda=D=\sigma=1$ and Ito calculus [16] has been used (different coefficients for the Laplacian and the KPZ non-linear term could be reabsorbed using $n=\exp (-\alpha h)$ with a proper choice of $\alpha$ ). This transformation maps the depinning from the wall $\langle h\rangle \rightarrow \infty$ to a transition into an absorbing state $\langle n\rangle \rightarrow 0$. The physical equivalence between the cases $\lambda>0$ with a lower-wall ( $p>0$ ), and $\lambda<0$ with an upper wall $(p<0)$, reflects in the fact that the same equation is obtained using $n=\exp (-h)$ and $n=\exp h$, respectively.

Observe that Eq.(2) is identical to the MN equation describing the MN1 class [2, 6, 7], except for the presence of an extra term $(\nabla n)^{2} / n$. This term can also be written as $(\nabla n) \cdot(\nabla \ln (n))=(\nabla n) \cdot(\nabla h)$ suggesting that the interface language is the natural one for this class. In fact, except for the factor 2 in front of $(\nabla n)^{2} / n$ Eq. (2) coincides with the Cole-Hopf transform of

$$
\partial_{t} h(x, t)=\nabla^{2} h+a+b e^{-p h}+\eta(x, t)
$$

that describes the growth of wetting layers toward their equilibrium state [19] (observe that this is just the equilibrium, Edwards-Wilkinson model, in the presence of a bounding wall). Note also that the factor 2 in Eq. (2) cannot be readsorbed by reparametrizing.

Finally, let us underline that in the regime where $b<0$ the wall becomes attractive (which might be necessary to describe some physical situations as, for instance, synchronization problems as said in the introduction) and a new term, say, $c \exp (-2 h)$ (equivalently $c n^{2 p+1}$ ) with $c>0$ has to be added to stabilize the equation.

Having presented the equations defining the model, in the forthcoming sections we study the associated physics by using i) mean field approaches, ii) field theory, and iii) numerical, Monte Carlo simulations combined with scaling arguments. 


\section{Mean-field approaches}

Mean-field approaches to Eq. (1) can be implemented with several degrees of sophistication. A crude approximation consists of ignoring the noise and spatial variations. At this level one trivially gets that the order parameter $\langle h\rangle$ vanishes as $a \rightarrow 0$ with an exponent $\beta_{h}=0$. When applied to Eq.(2), this approximation yields a shifted critical point at $a_{c}=-1$ and the usual result for the order parameter critical exponent, $\beta_{n}=1 / p$. But, as experience with other systems with multiplicative-noise dictates, neglecting completely the noise is a too crude approximation, that eliminates most of the characteristic traits of $\mathrm{MN}$ physics.

Now the effect of allowing a spatially varying order pa- rameter and taking the noise into consideration are examined. The Laplacian is discretized as

$$
\nabla^{2} n_{i}=\frac{1}{2 d} \sum_{j}\left(n_{j}-n_{i}\right) \approx\langle n\rangle-n_{i},
$$

where the sum runs over the nearest neighbors of $i$ and a large system dimensionality has been assumed. Similarly, the square gradient term can be written as

$$
\frac{(\nabla n)_{i}^{2}}{n_{i}} \approx \frac{\left\langle n^{2}\right\rangle}{n_{i}}-2\langle n\rangle+n_{i}
$$

The one-site stationary probability distribution is then readily obtained from the associated Fokker-Planck equation,

$$
\begin{aligned}
P_{s t}(n,\langle n\rangle) & \propto \frac{1}{n^{2}} \exp \int^{n} \frac{(\nabla x)_{i}^{2}-2(\nabla x)_{i}^{2} / x_{i}-(a+1) x_{i}-b x_{i}^{p+1}}{x_{i}^{2}} d x_{i}, \\
& \approx \frac{1}{n^{a+6}} \exp \left(-5 \frac{\langle n\rangle}{n}-b \frac{n^{p}}{p}+\frac{\left\langle n^{2}\right\rangle}{n^{2}}\right) .
\end{aligned}
$$

where $\langle n\rangle$ and $\left\langle n^{2}\right\rangle$ have to be calculated self-consistently. For $a<a_{c}=-5, P_{s t}$ is not normalizable, which means that the stationary state is the absorbing phase $\langle n\rangle=0$. For $\langle n\rangle \neq 0$, however, the non-analyticity of $\exp \left[\left\langle n^{2}\right\rangle / n^{2}\right]$ at $n=0$ again renders $P_{s t}$ non-normalizable. As a result, there is no well-defined active phase at this mean-field level for Eq. (6). Similar problems are found if the same type of approach is applied to Eq. (1) instead of Eq. (2).

To avoid the presence of the square gradient term in Eq. (2), and the complications of its, somewhat arbitrary, discretization, we resort to a different change of variables. After $n=\exp h$,

$$
\begin{aligned}
\partial_{t} n & =\nabla^{2} n+(a+1) n+b n^{3-p}+n \eta, \\
P_{s t} & \sim \frac{1}{n^{2-a}} \exp \left[\frac{b n^{2-p}}{2-p}-\frac{\langle n\rangle}{n}\right] .
\end{aligned}
$$

These equations are simpler than (2) and (6), but at the cost that $\langle n\rangle$ is no longer an order parameter as, at the transition point $\langle n\rangle \rightarrow \infty$ rather than going to 0 . Furthermore, Landau expansions only make sense when the order parameter vanishes at the critical point, making the whole approach inconsistent. One possible way to circumvent this problem is to monitor $m \equiv 1 / n$, and study $\tilde{P}_{s t}(m) d m=P_{s t}(n) d n$, but given the non-Gaussian nature of the probability distribution the substitution $\langle n\rangle=1 /\langle m\rangle$ is likely to be incorrect, and there seem to be no safe way to proceed.

Summing up, non-trivial mean-field approaches detect some problems with the model under consideration, and are not able to predict a phase correct phase diagram. A sound mean-field approximation therefore needs to be found. Notice that none of these problems occur in the case of a negative KPZ non-linearity, i.e. in the MN1 class, where a stan- dard mean-field approximation yields qualitatively correct results (see $[2,12]$ and references therein).

\section{Field theoretical considerations}

The Langevin equation for MN1 is known to be superrenormalizable, i.e. Feynman diagrams can be computed to all orders and resummed. This does not imply that critical exponents can be computed in all the cases, as the renormalization group flow-equation has runaway trajectories supposed to converge to a strong coupling fixed point. But at least, the correct phase diagram, including strong and weak coupling fixed points can be obtained.

For the MN2 the situation is far more complicated, as can be a priori anticipated given the failure of mean-field approaches. The extra term, $(\nabla n)^{2} / n$, being singular in $n$ precludes the use of perturbative expansions around $n=0$. Given the lack of a non-perturbative approach to the KPZ and MN strong coupling fixed points, there is not much we can add to this section, except that there is a promising attempt to tackle this and related KPZ-like problems. There is a formalism, developed by Fogedby, aimed at developing a strong coupling theory for KPZ based on a semiclassical or WKB approximation applied upon the Martin-Siggia-Rose generating functional [20]. Its main advantage is that it does not involve expansions around classical noiseless solutions, but around classical (extremal) noisy solutions. It would be very interesting to extend these ideas to $\mathrm{KPZ}$ problems in the presence walls, namely, to the multiplicative noise universality classes MN1 and MN2. 


\section{Numerical results}

Owing to the failure of standard (nontrivial) mean-field approaches and lacking so far an alternative analytical route, numerical methods are required to glean insight into the system properties. We have carried out simulations of two surface growth models. Both of them, in the absence of walls, are known to belong to the KPZ universality class. An extra rule is then added to generate a bounding wall, as described above.

\section{V.1 Model 1}

Our first model was introduced in [21] and is defined as follows: the surface position at time $t$ above a site $x$ on a one-dimensional lattice of size $L$ is given by a continuous height variable $h_{t}(x)$. A new height configuration is then generated in a three-step process.

1. Each lattice site $h_{t}(x)$ is updated according to $h_{t}^{\prime}(x)=h_{t}(x)+a+\eta_{t}(x) . \eta_{t}(x)$ is a random number uniformly distributed in $[0,1]$ and $a$ is a constant drift term analogous to that of (1).

2. The configuration is changed to $h_{t+1}(x)=$ $\min \left[h_{t}^{\prime}(x \pm 1)+\gamma, h_{t}^{\prime}(x)\right]$, where $\gamma$ is a constant whose precise value is not essential for the final results. We have set without loss of generality $\gamma=0.1$ as in previous numerical analyses.

3. A hard wall at $h=0$ is introduced by way of the additional rule $h_{t}(x)=\min \left[h_{t}(x), 0\right]$ [8].

Finally, periodic boundary conditions are imposed and $h_{t}(x)$ is initially set to 0 .

The continuum counterpart of this model is known to be a KPZ equation [21] with $\lambda<0$ in the presence of an upper wall [22] which, as remarked before, is equivalent to the case $\lambda>0$ and a lower wall, and corresponds to the MN2 class. Numerical results for this model were first presented in [8] and seemed to be consistent with mean-field (i.e. single-site) like behavior. However, a similar problem recently studied in the context of synchronization has revealed inconsistencies probably due to insufficient statistics $[2,13]$. In this subsection improved simulation results are provided upon revisiting the analysis reported in [8] for larger system sizes and longer sampling times. The case of an attractive wall, not included in [8], is also considered.

First, we take up the case of a simple (non-attractive) wall, corresponding to $b>0$ in Eqs. (1) and (2). Fig. (1) shows how the steady order parameter $\langle n\rangle$ changes with the system size $L$. Within the active phase, it saturates to a constant value, while in the absorbing one it bends down and decays exponentially. Our best estimate for the critical point is $a_{c}=1.57433(2)$ and from the slope of the curve we get $\beta_{n} / \nu_{\perp} \approx 0.33(2)$ [23]. This value of $a_{c}$ corresponds to the point where a free interface (far from the wall) has zero average velocity. The time evolution of $\langle n\rangle$ at the critical point for different system sizes (Fig. (2)) behaves like $\langle n(t)\rangle \sim t^{-\theta_{n}}$, with $\theta_{n}=0.215(15)$ or, equivalently, $\langle h(t)\rangle \sim t^{\theta_{h}}$, with $\theta_{h}=0.355(15)$. As for the exponents $\beta$, which govern the saturation of the order parameter within the active phase, they have been computed using the largest available system sizes $(L=1600$ and 3200$)$. The best fit to $\langle X\rangle \sim\left|a-a_{c}\right|^{ \pm \beta_{X}}$ yields $\beta_{n} \approx 0.32(3)$ (for $\langle n\rangle$ ) the and $\beta_{h}=0.52(2)$ (for $\langle h\rangle$ ), respectively. The error margin is typically larger here than for other exponents due to the sensitivity to the uncertainty in the determination of the critical point.

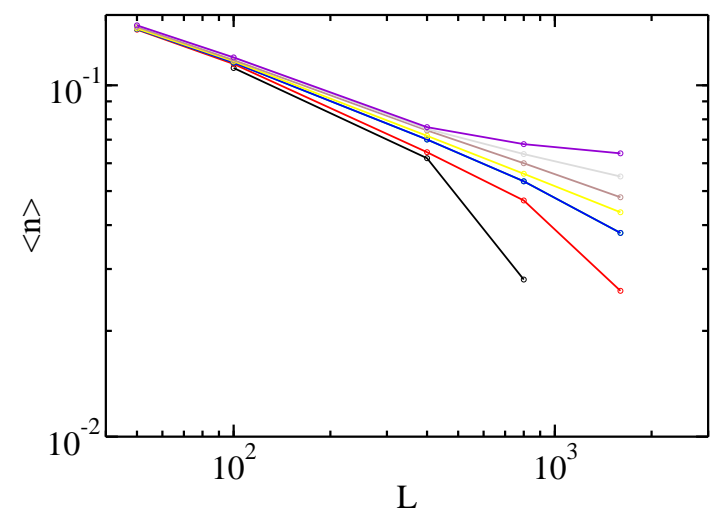

Figure 1. Model 1: steady-state values of the order parameter, $\langle n\rangle=\langle\exp h\rangle$, as function of the system size, $L$, for drifts (top to bottom) 1.57450, 1.57440, 1.57435, $1.57433,1.57430,1.57425,1.57420$. $\langle\cdot\rangle$ denotes both spatial and temporal averages, as well as averages over independent runs. The straight line corresponds to the critical point $a_{c}=1.57433(2)$ and from its slope $\beta_{n} / \nu_{\perp} \approx 0.33(2)$.

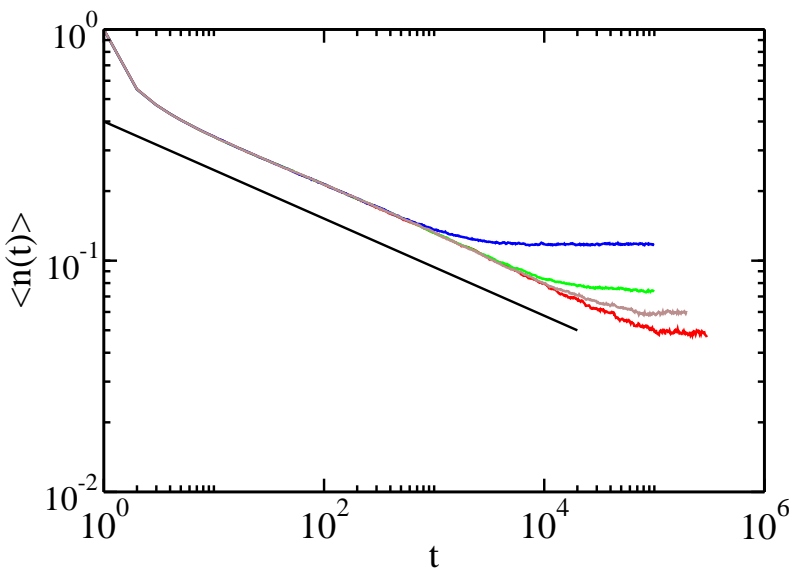

Figure 2. Model 1: time evolution of the order parameter, $\langle n(t)\rangle$, at criticality, $a=a_{c}$, for system sizes $100,400,800$, and 1600. The saturation values are those of Fig. 1. The best fit gives $\theta_{n}=0.215(15)$. The straight line is a guide for the eye and has a slope -0.215 .

It was proved in $[6,7]$ that these exponents must satisfy the scaling relation $z=\beta /\left(\nu_{\perp} \theta\right)$, where $z$ is the dynamic exponent of the KPZ equation. The exact value for $z$ in $d=1$ is $3 / 2$, thereby $z=0.33 / 0.215=1.5(1)$ in agreement with the prediction. In addition, also from $[6,7], \nu_{\perp}=1$, and from our direct measurements we get 
$\left(\beta_{n} / \nu_{\perp}\right) / \beta_{n}=0.33 / 0.32$, implying $\nu_{\perp} \approx 1$. In terms of $h$ and assuming $\nu_{\perp}=1, z=0.52 / .355=1.5(1)$ which, again, is compatible with $3 / 2$ within error bars.

The two alternative, but equivalent, mathematical descriptions of the MN2 class in terms of $h$ and $n=\exp (-h)$ can be related noting that the latter is essentially the density of sites at zero hight, $n(x, t)=\delta_{h(x, t), 0}$ [24]. We have verified that $n$ and $\delta_{h(x, t), 0}$ exhibit the same asymptotic scaling behavior.

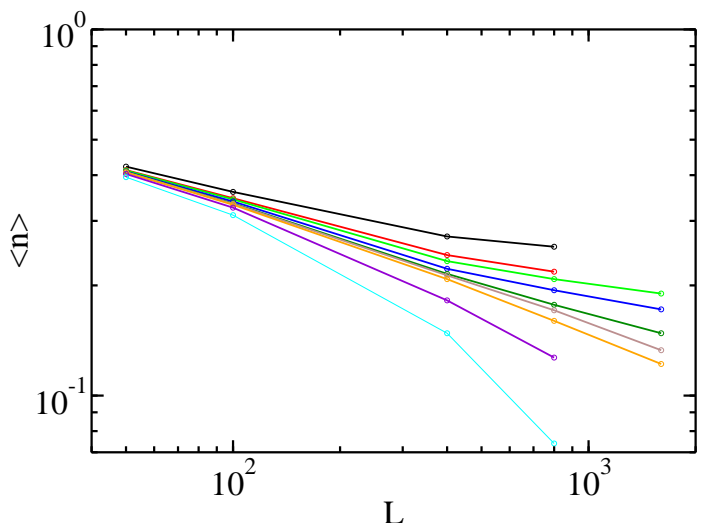

Figure 3. Model 1: steady-state values of the order parameter for an attractive wall, $b=-0.3$, as function of the system size $L$ for drifts (top to bottom) 1.57450, 1.57447, 1.57445, $1.57440,1.57435,1.57433,1.57430,1.57425,1.57420$. The best fit to a straight line again corresponds to $a_{c}=1.57433$.

Attractive walls can also be simulated within this model by simply replacing $a$ by $a-b \delta_{h, 0}$, where $b<0$ and the sign convention is chosen to keep the analogy with Eq. (1). This means that wherever the interface is attached to the wall it experiences an additional ("sticky") force pushing it against the wall. Extensive Monte Carlo simulations for $b=-0.3$ show that the previous results, in what respect universal features, carry over without change, the only difference being that the approach to asymptotics is slower. Upon increasing the attractiveness of the wall the transients become longer. The estimate for the critical point is the same as before (this is due to the fact that the free interface is not affected by variations of the attractiveness parameter). Again, our best estimates for the critical exponents are $\beta_{n} / \nu_{\perp}=0.32$ and $\theta_{n}=0.215$ (see Fig. (3)). For $b=-0.4$ we still observe a second-order phase transition with a crossover to the mentioned exponents. For $b=-0.5$ transition becomes firstorder but it still occurs at $a=a_{c}$. Within the active phase all the sites are close to the wall and the order parameter is 1 , but it suddenly changes to 0 upon decreasing $a$ and hysteresis is observed for slightly subcritical values $a$ (the interface is pinned up to long times). Therefore, a tricritical point must exist between 0.4 and 0.5 . We have identified it at $b=-0.42(1)$. The tricritical behavior has not been analyzed.

Let us stress that, contrary to what happens for the MN1 class, where the presence of an attractive wall induces a new and rich phenomenology (including a broad region of phase coexistence and directed-percolation type transitions [2]), the addition of "attractiveness" has a very mild effect here. Basically, it just shifts the position of the critical point and induces a first-order phase transition for very strong attractions.

\section{V.2 Model 2}

In order to verify the robustness and eventual universality of the previous results, we have performed a second study of a different model. It is a restricted solid-on-solid (RSOS) model, a variant of the single-step model introduced in [21], in the presence of a wall. A similar model has been recently studied in the context of synchronization transitions [25], to study MN1 type transitions. Initially the wall is located at $h_{w}=0$ and a grooved interface is placed beneath it, i.e. the interface has negative height at all the positions.

The dynamics proceeds as follows: At each time step, a site is randomly picked from a one-dimensional lattice of length $L$ and its height decreased two units, $h(i) \rightarrow h(i)-2$, provided that $h(i)>h(i+1)$ and $h(i)>h(i-1)$, i.e. provided that it is a local maximum. Should the the RSOS constraint be violated, the trial is discarded and repeated. Every $2(L-1) /\left[1-2 \delta v\left(1-L^{-1}\right)\right]$ steps the wall retreats one unit and, simultaneously, the interface is moved downwards by two units wherever it lies above the wall [25]. The difference between the wall and interface velocities, $\delta v$, acts as the control parameter: if $\delta v$ is negative, the interface eventually depins from the wall, while for $\delta v>0$ it remains pinned. It can easily be shown, using random walk arguments, that for the chosen wall velocity the system sits at its critical point. By varying it, we have a control parameter. The possibility of computing analytically the critical point largely simplifies the numerical analysis, and makes of this an efficient discrete model.
A

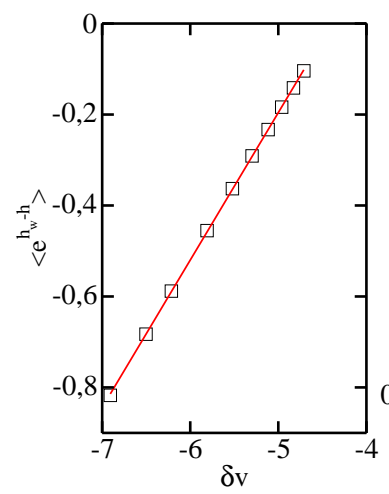

B

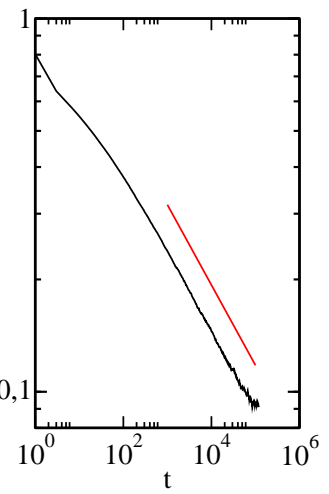

Figure 4. Model 2: (A) order parameter behavior, $\left\langle\exp \left(h_{w}-h\right)\right\rangle$, in the vicinity of the critical point $\delta v_{c}=0$. From the slope of the line we get $\beta_{n}=0.325(5)$. (B) Decay of the order parameter, $\left\langle\exp \left(h_{w}-h\right)\right\rangle$, at the critical point yields $\theta_{n}=0.215(5)$ (cf. with Fig. (2). The straight line is a guide for the eye and has a slope -0.215 .

The quantities monitored are $\left\langle\exp \left(h_{w}-h\right)\right\rangle$ and $h_{w}-h$. We have measured the exponents $\beta_{n}$ and $\theta_{n}$ for a system of $L=2^{20}$ sites. Our results lead to $\beta_{n}=0.325(5)$ and $\theta_{n}=0.215(5)$, in excellent agreement with those of model 
1 (Fig. (4)). Once more, assuming $\nu_{\perp}=1$, we get $z \approx 1.51$ in good agreement with the scaling laws. In addition, we have also measured the spreading exponent, $\eta$, that characterizes the number of pinned sites. It is computed averaging over all the runs and starting with an initial condition with a single point attached to the wall. Our best estimate is $\eta=0.80(2)$ (Fig. (5)). Measuring the survival probability, and therefore the exponents $\delta$ and $\zeta^{\prime}$, is a delicate technical point because it is hard to decide when the activity of a run has ceased. We have not tackled this problem here. Lastly, we obtain $\theta_{h}=0.34(5)$, which is again in good agreement with the value reported for our Model 1.

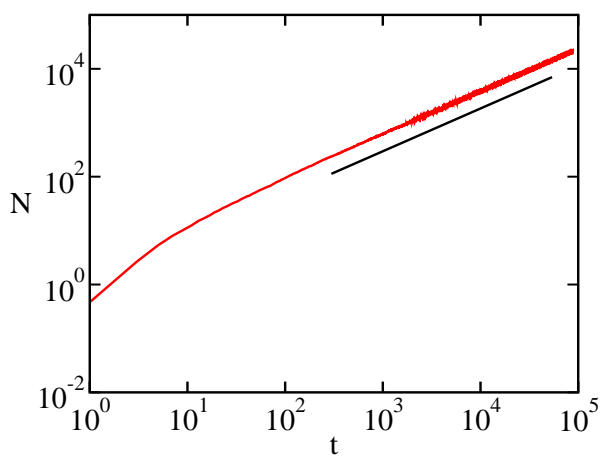

Figure 5. Model 2: number of pinned sites as a function of time for an initial condition with a single point attached to the wall. A fit for late times yields $\eta=0.80(2)$. The straight line is a guide for the eye and has slope 0.8 .

We have also considered different variations of the model in which the interface can penetrate the wall at some points, i.e. the wall is not perfectly rigid. None of the universal features seem to be affected by this change. In conclusion, all these results support strongly the existence of robust universality in the MN2 class.

As a matter of consistency, we have modified the algorithm of the model to simulate a lower wall, and therefore a case expected to be in the MN1 class. We obtain the set of exponents $\beta_{n}=1.69, \theta_{n}=1.19$ and $\eta=-0.4$, all of them in agreement we previously reported results and showing that the upper and lower problem belong to different universality classes $[2,7,9]$.

\section{V.3 Numerical integration of stochastic differ- ential equations}

As a further test for universality, we have numerically integrated Eq. (1) using Milshtein's algorithm [26]. A system size of $L=2000$ was considered and the time step and mesh size were set to $\Delta t=0.001$ and $\Delta x=1$, respectively. For simulation times up to $t=10^{6}\left(10^{9}\right.$ trials per site) our results lie far from the asymptotic regime. We do not discard numerical instabilities in the integration scheme, as it is known that results from numerical integrations may not agree with the predictions from the continuum KPZ [27]. The Cole-Hopf transform is a standard way to account numerically for the integration of bounded KPZ equations and, indeed, Langevin equations with $\mathrm{MN}$ are by far more stable than their interface-language KPZ-like counterparts [7, 9]. Nevertheless, we have also found numerical problems when integrating (2), either when the extra term is written in logarithmic form or averaging for smoothing the gradient. All numerical attempts are unstable nearby the absorbing state, owing to the presence of the extra singular term. We leave, therefore, the numerical integration of a continuous Langevin equation, representative of the MN2 class as an open, challenging problem.

\section{Discussion}

We have characterized the MN2 universality class, or analogously its bounded KPZ counterpart, which, as commented above, accommodates different physical phenomena. We have studied it from, somehow deceptive mean-field and field theoretical approaches, as well as by numerical studies. None of the analytical methods provides a satisfactory description of the phase transition present in this class. On the other hand, Monte Carlo simulations of two different discrete interface models, argued to belong to this universality class, give firm evidence for the existence of a robust universality class. Contrary to what previous simulations seemed to indicate [8], our results are not a simple extension of the ones obtained for one-site, implying that spatial correlations play an important role. Table 1 gathers the values of the critical exponents in terms of $n$ for the two discrete models considered in this paper. From the Monte Carlo estimates, it cannot be discarded that they adopt the rational values $\beta_{n}=1 / 3$ and $\theta_{n}=2 / 9$, which combined with the exact values derived in [6, 7], $\nu_{\perp}=1$ and $z=3 / 2$, would lead to $\beta_{n} / \nu_{\perp}=1 / 3$. Note that our results do not compare well with those of the nonequilibrium wetting model reported in [11]. We believe this is probably due to the extremely long transients known to be present in that model. We have verified that the transition point is located at the same value of the control parameter for any value of the "attractiveness" parameter ( $b$ in Model 1), either representing an attractive or a non attractive wall. For strong enough attractive walls, i.e. $b$ sufficiently negative, the transition becomes first order as in [11], while if the wall is weakly attractive it remains in the MN2 class.

\begin{tabular}{lcccccc} 
& $\beta_{n}$ & $\nu_{\perp}$ & $\beta_{n} / \nu_{\perp}$ & $z$ & $\theta_{n}$ & $\eta$ \\
\hline Model 1 & $0.32(2)$ & $0.97(5)$ & $0.34(2)$ & $1.55(5)$ & $0.215(15)$ & not measured \\
Model 2 & $0.325(5)$ & $\approx 1$ & $0.33(2)$ & $\approx 1.5$ & $0.215(5)$ & $\eta=0.8$ \\
\hline
\end{tabular}

Table 1. Critical exponents for the MN2 class in $d=1$. 
The problem of reaching a satisfactory analytical understanding, and even that of obtaining sound results from numerical integrations of the continuous Langevin-equation (in either the interface or the density language) representative of this class remains an open challenge.

Summing up, even though strong evidence is provided confirming the existence of a universality class (i.e. the corresponding critical exponents are computed with good precision in one dimension and they are universal in two different discrete models), its theoretical description in terms of Langevin equations, contrary to what happens for the closely related MN1 class, is far from satisfactory. In particular, the Langevin equation does not seem to admit sound mean-field solutions, nor is it amenable to being treated by means of standard perturbative field theoretical tools, nor does it admit stable numerical integration. Identifying the physical causes at the root of these difficulties is a challenge for future research.

\section{Acknowledgments}

F.S. acknowledges financial support from the Fundação para a Ciência e a Tecnologia, contract SFRH/BPD/5654/2001. Financial support from the Spanish MCyT (FEDER) under project BFM2001-2841, and from the AECI, are also acknowledged.

\section{References}

[1] See J. García-Ojalvo, and J. M. Sancho, Noise in Spatially Extended Systems, (Springer, New York, 1999); and references therein. See also, J. M. Sancho and J. García-Ojalvo, in Lecture Notes in Physics 557, p.235, edited by J. A. Freund and T. Pöschel, (Springer-Verlag, Berlin, 2000).

[2] M. A. Muñoz, preprint 2003, cond-mat/0303650.

[3] M. Kardar, G. Parisi and Y. C. Zhang, Phys. Rev. Lett. 56, 889 (1986).

[4] T. Halpin-Healy and Y.-C. Zhang, Phys. Rep. 254, 215 (1995); and references therein.

[5] A. L. Barabási, H. E. Stanley, Fractal Concepts in Surface Growth (Cambridge University Press, Cambridge, 1995); and references therein.

[6] G. Grinstein, M.A. Muñoz, and Y. Tu Phys. Rev. Lett. 76, 4376 (1996).

[7] Y. Tu, G. Grinstein and M.A. Muñoz, Phys. Rev. Lett. 78, 274 (1997).

[8] M.A. Muñoz and T. Hwa, Europhys. Lett. 41, 147 (1998).

[9] W. Genovese and M.A. Muñoz, Phys. Rev. E 60, 69 (1999).

[10] H. Hinrichsen, R. Livi, D. Mukamel, and A. Politi, Phys. Rev. Lett. 79, 2710 (1997).

[11] H. Hinrichsen, R. Livi, D. Mukamel, and A. Politi, Phys. Rev. E 61, R1032 (2000).
[12] F. de los Santos, M. M. Telo da Gama, and M. A. Muñoz, Europhys. Lett. 57, 803 (2002); Phys. Rev. E 67, 021607 (2003); Proceedings of the 7th Granada Seminar on Computational Physics. edited by J. Marro and P. L. Garrido; (American Institute of Physics, 2003), p 661; Cond-mat/0211124.

[13] M. A. Muñoz and R. Pastor Satorras, Phys. Rev. Lett. 90, 204101 (2003).

[14] T. Hwa and M. Lassig, Phys. Rev. Lett. 76, 2591 (1996). See also, T. Hwa and M. Lassig, "Optimal Detection of Sequence Similarity by Local Alignment" in Proceedings of the Second Annual Int. Conf. on Computational Molecular Biology (RECOMB98), edited by S. Istrail, P. Pevzner, and M.S. Waterman, 109-116 (ACM Press, 1998) and references therein; R. Olsen, T. Hwa and M. Lassig, "Optimizing Smith-Waterman Alignments" in Pacific Symposium on Biocomputing 4, 302313 (1999).

[15] R. Lipowsky and M.E. Fisher, Phys. Rev. B 36, 2126 (1987).

[16] The sole difference between utilizing the Ito or the Stratonovich conventions, in this case, is a trivial shift in $a$ $[17,18]$.

[17] N. G. van Kampen, Stochastic Processes in Physics and Chemistry, (North Holland, Amsterdam, 1981).

[18] C. W. Gardiner, Handbook of Stochastic Methods, (Springer Verlag, Berlin and Heidelberg, 1985).

[19] R. Lipowsky, J. Phys. A 18, L585 (1985).

[20] H. D. Fogedby, Phys. Rev. E. 57, 4943 (1998). H. D. Fogedby, Cond-mat/0303632.

[21] J. Krug, Adv. in Phys. 46, 139 (1997). J. Krug and H. Spohn, in Solids far from equilibrium, edited by C. Godrèche, (Cambridge University Press, Cambridge, 1991).

[22] Observe that the presence of the function "min" in the second step of the algorithm induces a negative average velocity, implying that in the continuum counterpart $\lambda$ has to be negative. On the other hand the last step $\min h(x, t), 0$ obviously generates an upper wall.

[23] We use the subscript $n$ to denote exponents related to the order parameter $n$ and ${ }_{h}$ for exponents associated with the average height.

[24] H. Hinrichsen, cond-mat/0302381.

[25] V. Ahlers and A. Pikovsky, Phys. Rev. Lett. 88, 254101 (2002). V. Ahlers, Ph. D. thesis. http://www.stat.physik.uni-potsdam.de/volker/publ.html. F. Ginelli et al., cond-mat/0302588.

[26] M. San Miguel and R. Toral, Stochastic Effects in Physical Systems, in Instabilities and Nonequilibrium Structures, VI, edited by E. Tirapegui and W. Zeller, (Kluwer Academic Pub., 1997). (Cond-mat/9707147).

[27] T. J. Newman and A. J. Bray, J. Phys. A 29, 7917 (1996). C.H. Lam and F. G. Shin, Phys. Rev. E 58, 5592 (1998). 